\section{Cryo Field Emission Scanning Electron Microscopy}

\section{Stanley L. Erlandsen, Cecile Ottenwaelter, Chris Frethem, and Ya Chen}

\author{
University of Minnesota School of Medicine, \\ Minneapolis, MN, USA
}

Low-temperature scanning electron microscopy (cryoSEM) was introduced in the early 1970s, shortly after the appearance of the first commercial scanning electron microscopes. This technique has been utilized widely in plant biology, the food and paper industry, X-ray analytical studies, and material sciences (8). Major advantages of cryoSEM include being able to avoid deleterious changes associated with preparative steps that remove water and produce extraction/shrinkage of tissue. CryoSEM can provide convenience and speed of sample preparation but has been considered to be a medium-resolution technique $(50-100 \mathrm{~nm})$. With the advent of field emission SEM (FESEM) and new developments in cryo-preservation of biological specimens, it has now become possible to achieve resolution in the macromolecular $(2-3 \mathrm{~nm})$ range.

\section{DEVELOPMENT OF FESEM}

The basic principles of SEM were developed in the 1930s coincident with the development of transmission electron microscopy (TEM). In conventional SEM (CSEM), resolution on biological specimens had been limited to $10-20 \mathrm{~nm}$ by a less efficient lens design and the use of a conventional $\mathrm{W}$ filament-based electron gun, which in turn limited beam brightness, electron probe diameter, and magnification, as well as by less than ideal specimen preparative methods. Accelerating voltages of 10-20 keV were commonly used in CSEM to achieve the small probe diameter needed for higher resolution. In the mid to late 1980 s, a number of improvements occurred in electron optical design that enabled FESEM to greatly exceed the resolution of CSEM $(9,16,17)$. These advances included the design of the immersion lens (where the specimen is inserted into the polepiece of the objective lens), the introduction of methods for producing 1-2 nm thick metal coatings, and the use of cold field emission electron guns that permitted high brightness with extremely small probe diameters (approximately $0.7 \mathrm{~nm}$ ).

In addition to these "in-lens" microscopes, a new class of "below-the-lens" microscope appeared in the mid 1990s that utilized a new "snorkel type" objective lens with improved optical design and reduced lens aberration coefficients. The electron probe diameter is one way to assess the potential resolving power of a scanning electron microscope in secondary electron (SE) imaging. In the Hitachi ${ }^{\circledR}$ S-900 cold field emission in-lens microscope (Hitachi Scientific Instruments, Mountain View, CA, USA), the probe size is 0.6-0.7 $\mathrm{nm}$ at $30 \mathrm{keV}, 1.2 \mathrm{~nm}$ at $3 \mathrm{keV}$, and $3 \mathrm{~nm}$ at $1.5 \mathrm{keV}$, although a new Hitachi model (S-5200) can achieve $1.8 \mathrm{~nm}$ at $1 \mathrm{keV}$. In the Hitachi S-4700 below-the-lens model, which is designed to handle biological samples up to $1 \mathrm{in}$. in diameter or thickness, a resolution on test specimens of $2.5 \mathrm{~nm}$ has been reported at $1 \mathrm{keV}$.

In addition to these optical developments, there have been more recent improvements, made by Autrata (1), in the design of yttrium aluminum garnet (YAG) scintillator detectors spiked with $\mathrm{Ce}$ for the detection of high take-off angle backscattered electrons (BSE) for high-resolution imaging. This YAG detector is highly sensitive, being able to discriminate less than 0.1 atomic number (at. no.), and the use of these detectors in either in-lens or below-the-lens field emission scanning electron microscopes greatly facilitates detection of small immunogold labels (Figure 1). Use of BSE imaging permits direct assessment of surface topography and detection of 3-12 nm colloidal gold labels by at. no. contrast in specimens coated with approximately $1 \mathrm{~nm}$ Pt to minimize charging in biological samples.

\section{SPECIMEN CRYO-PREPARATION}

To obtain molecular topographic information of cell structure with nanometer resolution, it is essential to preserve biological structure with a high degree of fidelity and not induce the formation of artifacts through preparative procedures. Conventional preparative methods for SEM, particularly dehydration and critical point drying, are satisfactory only for low resolution FESEM and are insufficient for investigation of macromolecular structure. Total removal of water from specimens causes molecular collapse or aggregation; therefore, bound water must be retained to maintain the integrity of macromolecular structure, and care must be taken in removing water from the biological sample (20). Controlled freezedrying differs from conventional freeze-drying in that the specimen temperature is not allowed to rise above $-85^{\circ} \mathrm{C}$, close to the temperature threshold for recrystallization of vitreous frozen water into ice type I (20). By not allowing the temperature to warm up above $-85^{\circ} \mathrm{C}$, the bound water associated with molecular surfaces is retained and molecular collapse is prevented. Through regulation of vacuum and the temperature of the specimen, ice can be sublimated from the specimen to expose the external surface of the cell membrane or macromolecules adsorbed onto a substratum without artifacts being introduced by ice crystal damage. Excellent results by cryoFESEM have been previously published using infinitely thin specimens (see below) of cryo-prepared bacteriophage $(11,12)$, reovirus $(2)$, fibronectin $(4)$, and actin $(3,9,25)$.

Cryo-preparation for FESEM imaging is a state-of-the-art technique and consists of four major preparative steps: cryoimmobilization, controlled freeze-drying, cryo-coating, and cryoFESEM observation. The most important step in cryopreparation of biological samples is to freeze the specimen to prevent damage from ice crystal formation that might distort the structure of the specimen. There are several fast-freeze methods, namely, plunge-freezing into an appropriate cryogen cooled to liquid nitrogen temperature, slam or impact freezing by direct contact of the specimen with a polished metal or jewel surface cooled to liquid nitrogen or liquid helium tem- 
perature, and propane jet freezing. With these rapid-freezing methods, the maximum thickness of the ice crystal-free layer is from 5 to $20 \mu \mathrm{m}$ for slam or plunge freezing. Although not as rapid as other freezing techniques, high-pressure freezing can cryo-immobilize cells within $8-10 \mathrm{~ms}$ and is performed at a pressure of greater than 2100 atm liquid nitrogen. The effect of high pressure is to reduce the freezing point of water from $0^{\circ} \mathrm{C}$ to $-23^{\circ} \mathrm{C}$ and also to inhibit the formation of type I ice crystals. Samples frozen by high-pressure freezing show vitrification of ice in the range of $400-500 \mu \mathrm{m}$, which is $20-50$ fold greater than rapid freezing.

Ideally, one would like to cryo-immobilize samples without any pretreatment. However, to examine cell surfaces with cryoFESEM, it is necessary to rinse the cell sample with distilled water; otherwise, buffer salts will precipitate and cover the sample when water is sublimated away under vacuum just before cryo-coating with metal. Examination of animal cells by cryoFESEM often requires that they be stabilized, such as pretreatment with glutaraldehyde before the isolation steps or immunolocalization of surface epitopes, then rinsed with distilled water immediately before rapid or high-pressure freezing.

\section{TYPES OF SPECIMENS FOR CryoFESEM}

The highest resolution of biological samples can only be obtained from an infinitely thin sample (<50 nm), and usually these specimens have been mounted on carbon support films on EM grids. Typical infinitely thin specimens used in highresolution studies have included viruses (2), polyheads of the bacteriophage T4 $(11,12)$, and the hexagonally packed intermediate (HPI) layer, a 2-D crystal found in the cell wall of the bacterium Deinococcus radiolarian (25). In these thin specimens, the use of high acceleration voltage electrons (20-30 $\mathrm{keV}$ ) makes the interaction volume very small, essentially making it transparent to the electron beam. The delocalized SE type 2 (SE2) signal that contributes to background is greatly reduced $(14,18)$. In addition, the probe size becomes smaller as the acceleration voltage is increased. A resolution of less than 2-3 $\mathrm{nm}$ has been reported on controlled freezedried, thin biological samples that are coated with $\mathrm{Cr}$ or $\mathrm{W}$ by

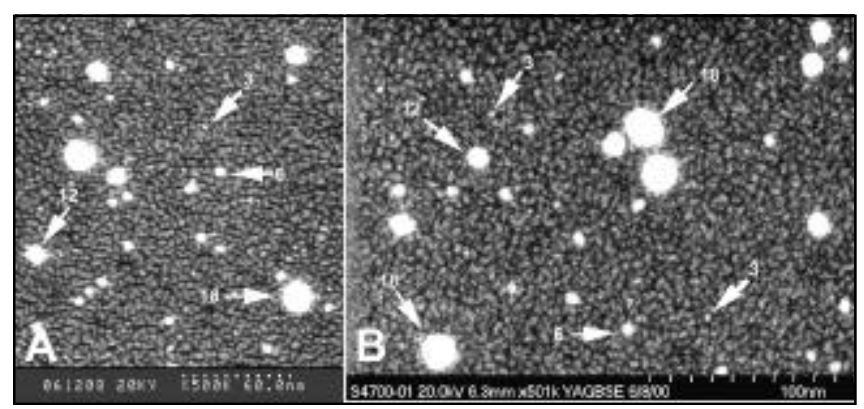

Figure 1. BSE images of a colloidal gold standard. High-resolution BSE images of a colloidal gold standard taken in (A) a Hitachi S-900 in-lens microscope or (B) a Hitachi S-4700 below-the-lens microscope, both of which are equipped with YAG BSE scintillator detectors. BSE images were collected at $20 \mathrm{keV}$ under ambient temperature and at magnification of $500 \mathrm{k} \times$. The colloidal gold specimen was coated with a thin $(<1 \mathrm{~nm})$ discontinuous layer of $\mathrm{Pt}$ by $\mathrm{Ar}^{+}$ion-beam sputtering. double-axis rotary shadowing (DARS) or planar-magnetron sputtering (PMS) at low temperature $(3,4,9,11,12,25)$. Illus trated in Figure 2 is a cryoFESEM image of actin filaments prepared as infinitely thin specimens and visualized by SE imaging. Individual subunits of actin are readily visible, and the helical repeats in the actin filament are also clearly seen.

High-resolution images can also be obtained from bulk samples that are coated with $\mathrm{Cr}$, W, or Pt $(3,13,22-24)$. Special attention must to be paid to imaging the surfaces of bulk samples because the bulk sample, due to its greater thickness $(>100 \mathrm{~nm})$, is not beam transparent.

If one wishes to use SE imaging on bulk samples, then the interaction volume of the beam in the specimen becomes important, and the low-resolution dislocated SE2 signal contributions cannot be ignored $(14,15,18)$. To reduce the contribution of SE2 signal, we need to choose the proper acceleration voltage. Higher voltage provides smaller probe size (improves resolution) but generates more SE2 signal and less contrast. Use of lower voltage $(<5 \mathrm{keV})$, often referred to as low-voltage SEM, provides the reverse, or higher contrast, and less SE2 signal due to less penetration of the primary electron beam. In examining bulk samples by SE imaging, a compromise is often necessary between the probe size and the ratio of SE1/SE2 generation. To minimize SE2 background, the BSE signal yield should be reduced by using $\mathrm{Cr}$ as the coating metal. Because $\mathrm{Cr}$ has a low at. no., fewer BSE are generated by interaction of the primary electron beam with $\mathrm{Cr}$, and this results in a higher ratio of SE1/SE2 and a reduction in background (11).

The double-layer coating technique for frozen-hydrated samples $(23,24)$ has been proposed to overcome issues related to radiation damage and contamination. The basic principle for double-layer coating is illustrated in Figure 3. A unidirectional shadow of a thin layer of heavy metal (e.g., Pt/C or $\mathrm{Ta} / \mathrm{W}$ ) is thermally evaporated onto the biological specimen surface, and then an additional 5-10 nm layer of $\mathrm{C}$ is added on top of the shadowed metal. The $\mathrm{C}$ coating enhances the mechanical stability of the specimen (22), thereby significantly reducing radiation damage and mass loss. Imaging of specimen topography is actually accomplished with the BSE sig-

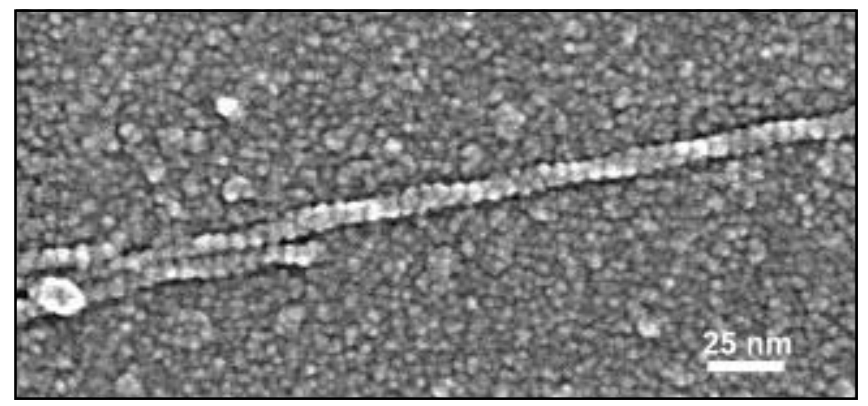

Figure 2. High-resolution secondary electron cryo-image of partially frozen hydrated actin filaments polymerized in vitro. These actin filaments were reconstituted in vitro and were stabilized with $1 \%$ glutaraldehyde, followed by a rinse with distilled water before cryo-immobilization in liquid propane cooled with liquid nitrogen. Frozen vitrified water was removed by sublimation, exposing the partially hydrated actin filament. The sample was cryo-coated with $\mathrm{Cr}$ at $-110^{\circ} \mathrm{C}$ by PMS, cryo-transferred in a Gatan cryostage into a Hitachi S-900 in-lens microscope, and examined at $-125^{\circ} \mathrm{C}$. 


\section{Bielmaging}

nal. The BSE signal is proportional to at. no.; therefore, it is generated from the metal coating but not the lighter $\mathrm{C}$ layer. The SE signal from these samples represents the surface of a thick $\mathrm{C}$ coating layer that is on top of the metal layer, not the metal-coated surface of specimen topography because lowenergy SE1 $(<20 \mathrm{keV})$ signals from the metal layer cannot penetrate the thick overlying $C$ layer. Using the double-coating method, Walther et al. $(23,24)$, in studies on membranes of fractured yeast, have demonstrated $10-\mathrm{nm}$ particles containing pores and a smaller class of 5-nm particles. In these studies, the resolution obtained was comparable to the freezefracture replica method in TEM. Recently, Erlandsen et al. $(9,10)$ used the double-layer coating method on bulk samples to reveal the individual molecular topography of three different cell adhesion molecules (CAMs) in the glycocalyx of spread human platelets. Specific antibodies to these three CAMs and immunogold markers were used to detect and identify each CAM. Figure 4 illustrates a cryo-image of a partially hydrated platelet, and the rod-shaped CAM, P-selectin, was visualized in the glycocalyx as projecting up above the membrane of activated human platelets. Up-regulation of Pselectin onto the platelet surface and its expression as dimers on the surface of the spread platelet membrane were accomplished by the release of this CAM from $\alpha$ granules $(9,21)$. As shown in Figure 5, the rod-shaped P-selectin molecule can be seen projecting approximately $45 \mathrm{~nm}$ above the surface of the membrane. The terminal lectin domain of P-selectin was im-

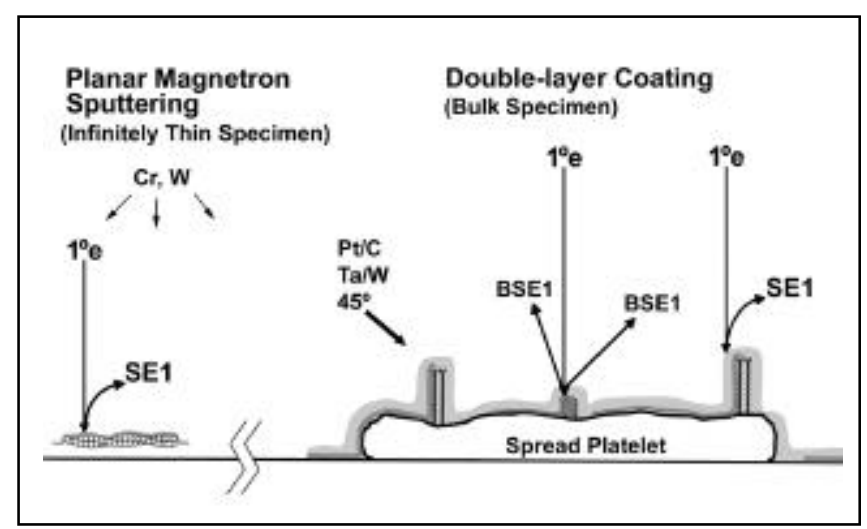

Figure 3. Diagram illustrating metal cryo-coating. Metal cryo-coating by (A) PMS of infinitely thin specimens and (B) the double-coating method of Walther et al. (23) for bulk specimens. The actin filament shown on the left was cryo-coated with either $\mathrm{Cr}$ or W by PMS. The primary electron beam $\left(1^{\circ} \mathrm{e}\right)$ impacts the metal coating, and high-resolution SE1 electrons are emitted and collected for image formation. On the right, a spread human platelet, with several P-selectin dimers projecting above its surface, has been cryo-immobillzed. The free frozen water was removed by sublimation to expose the partially hydrated P-selectin molecules on the membrane surface. The specimen was then unidirectionally coated with approximately $2 \mathrm{~nm}$ either $\mathrm{Pt} / \mathrm{C}$ or $\mathrm{Ta} / \mathrm{W}$ at a $45^{\circ}$ angle, followed by an additional layer of $\mathrm{C}$ at $90^{\circ}$ to stabilize the metal coating. When the $1^{\circ} \mathrm{e}$ strikes the $\mathrm{C}$ layer and gives rise to the SE1 signal, these electrons do not contain topographical information about the platelet surface because they were generated from the surface of the supporting $\mathrm{C}$ layer, and their low energy level does not excite the BSE detector. The $1^{\circ} \mathrm{e}$ also penetrates the $\mathrm{C}$ layer, which strikes the metal layer, giving rise to high-energy BSE1 signals that penetrate the $\mathrm{C}$ layer and excite the YAG BSE detector. The BSE1 signals provide topographical information on the metal coating of extracellular molecules on the platelet membrane and can provide at. no. contrast for detection of colloidal gold probes. munolabeled with $10 \mathrm{~nm}$ colloidal gold, which appears as white spheres by BSE imaging, and careful examination of the membrane between the P-selectin molecules reveals small knob-like elevations that have been shown to represent integrins on the platelet membrane (9).

\section{IMPORTANCE OF METAL COATING}

Metal coating of specimens is a critical step for increasing signal yield, enhancing contrast, and reducing charge generation $(6,15,17,19)$. Biological samples normally have an insufficient SE signal yield and a poor signal-to-noise ratio because of their low at. nos. and poor electrical conductivity. A thin semi-continuous metal film of uniform thickness is necessary to generate the high-resolution SE1 or BSE1 signal at the specimen surface. To achieve a high-quality metal coating, it is important to control metal thickness, grain size, geographic distribution of the metal particle, and to choose the proper metal to maximize SE and BSE signal generation and imageresolving power (7). Different metals for high-resolution SEM have been investigated in detail during the past decade, and $\mathrm{Cr}, \mathrm{W}, \mathrm{Pt}$, and $\mathrm{Pt} / \mathrm{Ir} / \mathrm{C}$ are examples of metals that have been applied in a variety of applications with different resolution requirements $(3,4,11,12,23,25)$. The ultimate resolution attainable with each metal is dependent on the metal properties, coating conditions, and selection of the appropriate signal mode for electron imaging. Fine grain size can be obtained using ion-beam sputtering (5), PMS (12), electron beam shadowing (25), and DARS (11). The grain size can be further decreased when the metal is coated at low temperature $(7,19)$.

The "classical resolution" in SEM as defined by Gabor is limited by the electron beam probe size and the beam-specimen interaction volume $(14,15)$. For high-resolution SEM by SE imaging, it is essential that the metal coating consist of fine grains so that signal generation is confined to the metal layer. The metal layer selected should have a small yield of BSE to reduce the production of SE2 signals, whose presence can degrade the high-resolution information contained in the SE1 signal. Joy (14) suggested applying a thin-layer metal film $(<2$ $\mathrm{nm}$ ) onto the low at. no. specimen to achieve resolution higher than the "classical limit". Thin uniform metal films with a

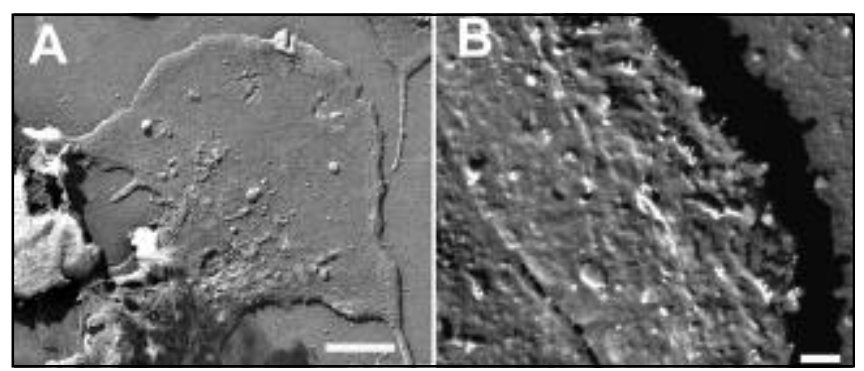

Figure 4. CryoFESEM images of spread human platelets. Platelets were immunolabeled with $10 \mathrm{~nm}$ gold for localization of P-selectin. (A) Low-magnification BSE image of a spread human platelet attached to the substratum. Bar equals $1 \mu \mathrm{m}$. (B) Higher-magnification BSE image of the edge of the same spread human platelet. P-selectin molecules are immunolabeled with $10 \mathrm{~nm}$ colloidal gold (white spheres). The shadow of the platelet is seen on the substratum on the right side of the image. Bar equals $200 \mathrm{~nm}$. 


\section{Bieolmaging}

thickness smaller than the SE-escape depth (1-2 nm for metals) are supposed to reveal a high-resolution topographic signal (14). The SE1 signal is generated primarily from the thin metal film. At the edge of the feature where the projected metal thickness increases, the SE1 signal yield increases accordingly. The width of the SE signal edge profile is similar to the thickness of the metal film. Theoretically, it should be possible to push the resolution power to less than $1 \mathrm{~nm}$ if an ultrathin (e.g., $0.5 \mathrm{~nm}$ ) layer of metal can be evenly applied (14).

To perform high-resolution BSE imaging, it is necessary to coat the specimen with a thin layer of metals, such as W, $\mathrm{Ta} / \mathrm{W}$, or Pt to obtain a high BSE1 signal. To localize colloidal gold probes (at. no. 79), W or Ta/W (at. nos. 73 and 74) provides better contrast than $\mathrm{Pt}$ (at. no. 78). Because of their lower at. no., the background is reduced with $\mathrm{W}$ or Ta/W coatings. Theoretically, thin metal coatings should yield high-resolution topographical images at the molecular level, but practically, determination of the optimal thickness of the metal coating may require compromising the signal-to-noise ratio because generation of the BSE signal is known to diminish with thinner metal coatings.

To obtain nanometer resolution, specimens must be imaged at very high magnifications $(>200 \mathrm{k} \times)$. Unfortunately, the beam damage or mass loss due to electron irradiation can be a serious limitation at high magnification. The electron dose at $100 \mathrm{k} \times$ magnification is approximately 2500 electron dose $/ \mathrm{nm}^{2}$ but increases by a factor of 16 as magnification increases by a factor of 4 (e.g., electron dose increases to 40000 electron dose $/ \mathrm{nm}^{2}$ at $400 \mathrm{k} \times$ ). Lowering the sample temperature will help reduce the rate of damage caused by irradiation but cannot totally eliminate it. Experimental data indicate that the thin metal coating does not prevent the underlying biological sample from beam damage (13).

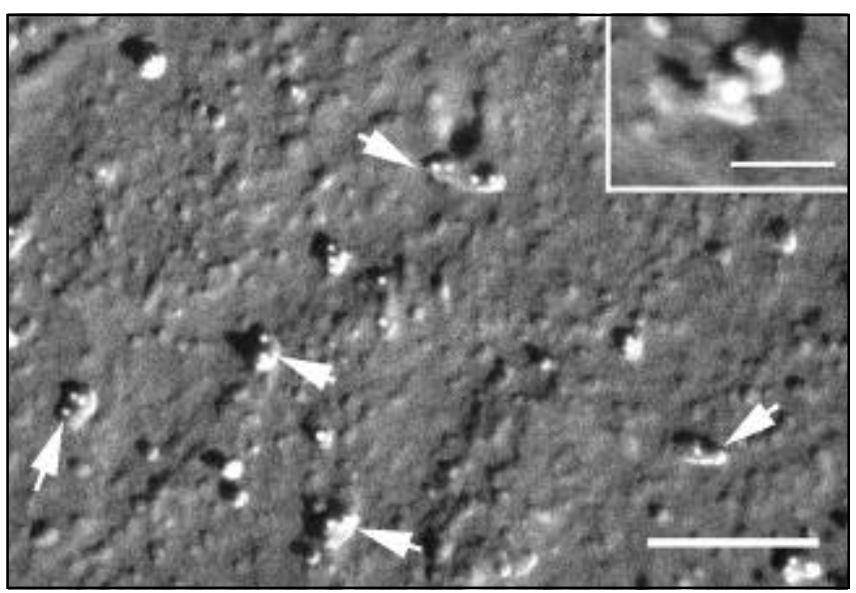

Figure 5. High-magnification cryoFESEM of membranes from spread human platelets. Platelets were immunolabeled with $10 \mathrm{~nm}$ colloidal gold (white spheres) for P-selectin. Dimers of P-selectin (arrows) can be seen as projecting above the membrane surface. Small knob-like protrusions in the background between P-selectin molecules resemble integrin molecules. Bar equals $200 \mathrm{~nm}$. Inset: Example of P-selectin dimer showing separate molecules and labeling of C-lectin domain with $10 \mathrm{~nm}$ colloidal gold (white spheres). Long shadows seen at the top of the molecules indicates their projection above the membrane surface, which was measured at approximately $45 \mathrm{~nm}$ by stereo imaging using the Sterecon computer program. Bar equals $50 \mathrm{~nm}$. (Reproduced by permission from Reference 10.)

\section{CONCLUSIONS}

The development and refinement of instrumentation, as well as progress in cryoFESEM, have ushered in a new era of the application of SEM to visualize surface features of biological specimens at high resolution. The use of fine, smalldiameter electron probes $(<1 \mathrm{~nm})$ permits easy and direct imaging of three-dimensional structure of biological surfaces with a resolution approaching that of TEM. Examination of cryo-specimens in combination with immunocytochemical localization of surface epitopes and molecular topography should facilitate a better understanding of cellular surfaces, the arrangement, distribution, and interrelationships of surface molecules, and their interactions with the environment. Continued improvement in metal coating technology and further development in producing smaller electron probes may help provide information on molecular definition and detail on intact biological samples that was previously considered impossible to obtain.

For more information on FESEM and cryoFESEM, see http://www.em.biol.ethz.ch/.

\section{ACKNOWLEDGMENTS}

We would like to thank the Graduate School at the University of Minnesota and the Minnesota Medical Foundation and National Institutes of Health grant no. R21 GM 59061 for partial financial support. We would like to acknowledge Dr. Anne Greet Bittermann for cryoFESEM work on human platelets, and Dr. Carol Wells for thoughtful suggestions and comments.

\section{REFERENCES}

1.Autrata, R. 1992. Single crystal detector suitable for high resolution scanning electron microscopy. EMSA Bull. 22:54-58.

2.Centonze, V.E., Y. Chen, G.G. Borisy, and M.L. Nibert. 1995. Visualization of individual reovirus particles by low-temperature, high-resolution scanning electron microscopy. J. Struct. Biol. 115:215-225.

3.Chen, Y., V.E. Centonze, A. Verkhovsky, and G.G. Borisy. 1995. Imaging of cytoskeletal elements by low temperature high resolution scanning electron microscopy. J. Microsc. 179:67-76.

4. Chen, Y., L. Zardi, and D.M. Peters. 1997. High-resolution cryo-scanning electron microscopy study of the macromolecular structure of fibronectin fibrils. Scanning 19:349-355.

5.Chopra, K.L. 1966. Growth of sputtered versus evaporated metal films. J. Appl. Phys. 37:3405-3410.

6. Echlin, P. and G. Kaye. 1979. Thin films for high resolution conventional scanning electron microscopy. Scanning Electron Microsc. II:21-30.

7.Echlin, P. 1981. Recent advances in specimen coating techniques. Scanning Electron Microsc. I:71-90.

8.Echlin, P. 1992. Low-Temperature Microscopy and Analysis. Plenum Press, New York.

9.Erlandsen, S.L., C. Frethem, and Y. Chen. 2000. Field emission scanning electron microscopy (FESEM) entering the 21st century: nanometer resolution and molecular topography of cell structure. J. Histotech. 23:249-259.

10.Erlandsen, S.L. A.G. Bittermann, J. White, A.D. Leith, and M. Marko. 2001. High resolution cryoFESEM of individual cell adhesion molecules (CAM) in the glycocalyx of human platelets: detection of P-selectin (CD62P), GPI-IX complex (CD42a/CD42b $\alpha, b \beta)$, and integrin GPIIbIIIa (CD41/CD61) by immunogold labeling and stereo imaging. J. Histochem. Cytochem. 49:809-819. 
11.Hermann, R., J.B. Pawley, T. Nagatani, and M. Müller. 1988. Doubleaxis rotary shadowing for high-resolution scanning electron microscopy. Scanning Microsc. 2:1215-1230.

12.Hermann, R. and M. Müller. 1991. Prerequisites of high resolution scanning electron microscopy. Scanning Microsc. 5:653-664.

13.Hermann, R. and M. Müller. 1993. Progress in scanning electron microscopy of frozen-hydrated biological specimens. Scanning Microsc. 7:343-350.

14.Joy, D.C. 1995. Limits of SEM resolution. Hitachi Instrument News 27:16-19.

15.Joy, D.C. and J.B. Pawley. 1992. High-resolution scanning electron microscopy. Ultramicroscopy 47:80-100.

16.Nagatani, T. and S. Saito. 1987. Development of an ultra high resolution scanning electron microscope by means of a field emission source and inlens system. Scanning Microsc. 1:901-909.

17.Pawley, J.B. 1997. The development of field-emission scanning electron microscopy for imaging biological surfaces. Scanning 19:324-336.

18.Pawley J.B. and S.L. Erlandsen. 1989. The case for low voltage high resolution scanning electron microscopy of biological samples, p. 163173. In R.M. Albrecht and R.M. Orenberg (Eds.). The Science of Biological Specimen Preparation for Microscopy and Microanalysis. Scanning Microscopy International, Chicago, IL.

19.Peters, K.R. 1986. Rationale for the application of thin, continuous metal films in high magnification electron microscopy. J. Microsc. 142:25-34.

20.Steinbrecht, R.A. and K. Zierold. 1987. Cryotechniques in Biological Electron Microscopy. Springer Verlag, New York.

21.Ushiyama, S., T.M. Laue, K.L. Moore, H.P. Erickson, and R.P. McEver. 1994. Structural and functional characterization of monomeric soluble P-selectin and comparison with membrane P-selectin. J. Biol. Chem. 268:15229-15237.

22.Walther, P. and J. Henschel. 1989 Improved representation of cell surface structures by freeze substitution and backscattered electron imaging. Scanning Microsc. Suppl. 3:201-211.

23.Walther, P., E. Wehrli, R. Hermann, and M. Müller. 1995. Double layer coating for high resolution low temperature SEM. J. Microsc. 179:229237.

24.Walther, P. and M. Müller. 1997. Double-layer coating for field-emission cryo-scanning electron microscopy-present state and applications. Scanning 19:343-348.

25.Wepf, R., U. Aebi, A. Bremer, M. Halder, P. Tittmann, J. Zach, and H. Gross. 1994. High-resolution SEM of biological macromolecular complexes, p. 1026-1027. In G.W. Bailey and A.J. Reed (Eds.). Proceedings of the 52nd Annual Meeting of the Microscopy Society of America. San Francisco Press, San Francisco, CA.

Address correspondence to Dr. Stanley Erlandsen, Department of Genetics, Cell Biology, and Development, 6-160 Jackson Hall, 321 Church St. S.E., University of Minnesota School of Medicine, Minneapolis, MN 55455, USA.e-mail: erlan001@ umn.edu

Suggestions for contributions to the Biolmaging feature are welcomed by its editor, Dr. Steve Paddock (paddock@facstaff.wisc.edu)

For reprints of this or any other article, contact Reprints@BioTechniques.com 